\title{
PRIMER REGISTRO DE UN CASO DE MALFORMACIONES EN LITHOBATES NEOVOLCANICUS (HILLIS \& FROST 1985) (ANURA: RANIDAE)
}

\author{
Barragán-Ramírez, J. L. \& J. L. Navarrete-Heredia. 2011. First record of limb malformations in \\ Lithobates neovolcanicus (Hillis \& Frost 1985) (Anura: Ranidae). Acta Zoológica Mexicana (n. s.), \\ 27(3): 837-841.
}

ABSTRACT. On September 9th, 2010, we found an individual of Lithobates neovolcanicus in a pond near to a disturbed pine forest from Mezcala de la Asunción, Poncitlán, Jalisco. The specimen presented two different deformities in its left forelimb and right hindlimb, polymelia and ectromelia, respectively. This is the first record of limb malformations in this endemic Mexican species.

Lithobates neovolcanicus (Hillis \& Frost 1985) es una especie endémica de México que habita en áreas abiertas de bosque de pino-encino y mezquite-pastizal, en elevaciones de 1500 a $2500 \mathrm{~m}$ sobre el nivel del mar ( $\mathrm{msnm})$ que presentan cuerpos de agua como lagos, estanques y arroyos. Se ha registrado en los estados de Aguascalientes (Vázquez-Díaz \& Quintero-Díaz 2005), Guanajuato, Michoacán (Hillis \& Frost 1985, Flores-Villela \& Canseco-Márquez 2007), Jalisco (Hillis \& Frost 1985, Flores-Villela \& Canseco-Márquez 2007, Reyna-Bustos et al. 2007), Zacatecas (Ahumada-Carrillo 2010) y México (Aguilar-Miguel et al. 2009). Es una especie que se encuentra enlistada en la NOM-059-SEMARNAT-2010 (SEMARNAT 2010), con categoría (A) Amenazada y en la lista roja de especies amenazadas de la IUCN con categoría (NT) "Near Threatened" (casi amenazada). Además de registros de distribución (ver citas arriba), existen otros trabajos sobre taxonomía y sistemática (ver Frost 2011) y sobre la dieta de este anuro (González-Hernández et al. 2010).

El incremento en la detección de malformaciones en anfibios ha generado un interés científico con la finalidad de conocer sus etiologías. Algunos factores que han sido reconocidos como causantes de malformaciones son: parásitos, como el helminto tremátodo Ribeiroia ondatrae (Johnson et al. 2001, Johnson et al. 2002, Ankley

Recibido: 26/10/2010; aceptado: 29/03/2011. 
et al. 2004, Johnson \& Lunde 2005) y otros Trematoda (Blaustein \& Johnson 2003, Rajakaruna et al. 2008), contaminantes ambientales como los agroquímicos (Blaustein \& Johnson 2003, Ankley et al. 2004, Robles-Mendoza et al. 2009), depredación selectiva (Gray \& Lethaby 2010), endogamia (Williams et al.2008) y altos niveles de radiación ultravioleta (Blaustein \& Johnson 2003, Ankley et al. 2004). Sin embargo, algunos de estos factores han sido cuestionados como posibles agentes causantes de malformaciones en anfibios, tal es el caso de los agroquímicos (Piha et al. 2006).

En México son pocos los trabajos publicados con información sobre estas malformaciones, aún cuando algunos agentes etiológicos son comunes. Una búsqueda en ISI Web of Science (Thomson Reuters 2010) utilizando como palabras clave: malformations, morphological abnormalities, deformities, Amphibia y México, generó sólo un registro de Ambystoma mexicanum (Robles-Mendoza et al. 2009) en donde se mencionan malformaciones en la cola de las larvas. Otro registro proviene de $\mathrm{Am}$ bystoma velasci en el cual se reporta braquidactilia y/o ectrodactilia y polidactilia (Cruz-Pérez et al. 2009).

El presente trabajo se deriva de la recolecta de un individuo juvenil de L. neovolcanicus, durante una salida a campo efectuada el 9 de septiembre del 2010 en Mezcala de la Asunción, Poncitlán, Jalisco. El ejemplar con una longitud total (LT) de $38 \mathrm{~mm}$ se encontraba en un estanque cercano a un bosque de pino perturbado por la actividad pecuaria y tala de árboles $\left(20^{\circ} 21^{\prime} 17.00^{\prime}{ }^{\prime} \mathrm{N}, 103^{\circ} 02^{\prime} 26.92^{\prime}{ }^{\prime} \mathrm{O}\right)$, a 1876 $\mathrm{msnm}$. Una vez examinado y fotografiado el ejemplar evidenciando las malformaciones apendiculares, fue liberado. Las imágenes tomadas originalmente a color fueron transformadas a blanco y negro, ajustando el contraste de manera automática utilizando el programa Corel Photo-Paint ${ }^{\circledR}$. Copias de las fotografías fueron depositadas

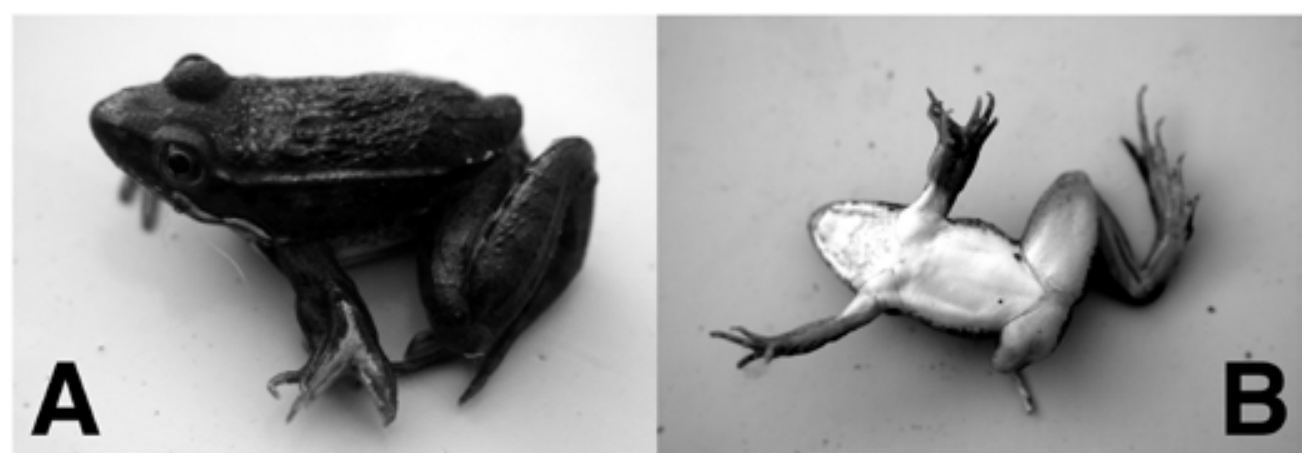

Figura 1. Lithobates neovolcanicus, espécimen examinado. A. Vista lateral mostrando polimelia en el miembro anterior izquierdo (MZFCID-20, CZUG-152). B. Vista ventral mostrando polimelia en el miembro anterior izquierdo y ectromelia de fémur en el miembro posterior derecho (MZFCID-22, CZUG-153). 
en la Colección Herpetológica del Museo de Zoología Alfonso L. Herrera, Facultad de Ciencias, UNAM (MZFCID) y en la Colección de Vertebrados del Centro de Estudios en Zoología, Universidad de Guadalajara (CZUG).

Durante la revisión del ejemplar se encontró que presentaba anomalías en dos de sus cuatro extremidades. El miembro anterior izquierdo (MAI) presentaba unido un miembro adicional apenas diferenciable en el que se reconocen claramente las estructuras de la mano, así como un notorio ensanchamiento en la región radio-ulnar que sugiere la duplicidad de estas estructuras. El miembro posterior derecho (MPD) presentó ausencia de estructuras en la parte inferior, terminando en la región mediadistal del fémur (Fig. 1).

Con base en los trabajos de Meteyer et al. (2000) y Meteyer (2000) se determinó el tipo de anomalía presente en cada uno de los miembros afectados, y se verificó que se trataba de malformaciones y no de daños por traumatismo (en el caso del MPD). La malformación de MAI se identificó como polimelia, mientras que la del MPD correspondió a ectromelia de fémur.

Se carece de evidencias científicas para explicar el origen de las malformaciones observadas en el espécimen así como de su incidencia en la población de L. neovolcanicus en la localidad, por ello, es conveniente incrementar el trabajo de campo en la región que implique muestreo de organismos y un análisis de las posibles causas implicadas en la aparición de malformaciones en ésta y otras especies de anfibios.

AGRADECIMIENTOS. A José de Jesús Ascencio Arraiga por su apoyo durante la colecta del ejemplar. A dos revisores anónimos por sus valiosos comentarios que permitieron modificar y mejorar el trabajo. A los curadores Oscar Flores Villela y Edmundo Pérez Ramos de la Colección Herpetológica del Museo de Zoología Alfonso L. Herrera (UNAM), y a Sergio Guerrero Vázquez y Daniel Cruz Sáenz de la Colección de Vertebrados del Centro de Estudios en Zoología (Universidad de Guadalajara) que brindaron su apoyo durante la incorporación del material fotográfico. De manera especial a Chris Grünwald por su ayuda para la determinación del ejemplar y a Miguel Vásquez Bolaños por sus comentarios al manuscrito.

\section{LITERATURA CITADA}

Aguilar-Miguel, X., G. Casas-Andreu, P. J. Cárdenas-Ramos \& E. Cantellano de Rosas. 2009. Análisis espacial y conservación de los anfibios y reptiles del Estado de México. Ciencia Ergo Sum, 16: 171-180.

Ahumada-Carrillo, I. T. 2010. Herpetofauna del Municipio de Atolinga, Zacatecas. Tesis Profesional, División de Ciencias Biológicas y Ambientales, Universidad de Guadalajara, Guadalajara, Jalisco.

Ankley, G. T., S. J. Degitz, S. A. Diamond \& J. E. Tietge. 2004. Assessment of environmental stressors potentially responsible for malformations in North American anuran amphibians. Ecotoxicology and Environmental Safety, 58: 7-16.

Blaustein, A. R. \& P. T. J. Johnson. 2003. The complexity of deformed amphibians. Frontiers in Ecology and Environment, 1: 87-94. 
Cruz-Pérez, M. S., J. A. Rangel-Hernández, O. Roldan-Padron, G. A. Soto-Alonso, U. Padilla-García \& U. O. García-Vázquez. 2009. Presencia de malformaciones en Ambystoma velasci en Alameda del Rincón, Querétaro, México. Boletín de la Sociedad Herpetológica Mexicana, 17: 92-96.

IUCN. 2010. IUCN Red list of threatened species. Version 2010.3. http://www.iucnredlist.org (Consultada el 7 de octubre de 2010).

Flores-Villela, O. \& L. Canseco-Márquez. 2007. Riqueza de la herpetofauna, pp. 407-420. In: Luna, I., J. J. Morrone \& D. Espinosa (Eds.). Biodiversidad de la Faja Volcánica Transmexicana. Universidad Nacional Autónoma de México. México, D.F.

Frost, D. R. 2011. Amphibian Species of the World: an online reference. Version 5.5 (31 January, 2011). American Museum of Natural History, New York, USA. Electronic Database accessible at http://www.research,amnh.org/vz/herpetology/amphibia/ (consultada el 24 de febrero de 2011).

González-Hernández, A. J., S. Reyes-Sosa \& V. H. Reynoso. 2010. Lithobates neovolcanicus: Diet. Herpetological Review, 41: 198.

Gray, B. S. \& M. Lethaby. 2010. Observations of limb abnormalities in amphibians from Erie County, Pennsylvania. Journal of Kansas Herpetology, 35: 14-16.

Hillis, D. M. \& J. S. Frost. 1985. Three new species of Leopard Frogs (Rana pipiens Complex) from The Mexican Plateau. Occasional Papers of the Museum of Natural History University of Kansas, 117: 1-14.

Johnson, P. T. J. \& K. B. Lunde. 2005. Parasite infection and limb malformations: A growing problem in amphibian conservation, pp. 124-138. In: M. J. Lannoo (Ed.). Amphibian declines: the conservation status of United States species. University of California Press. Berkeley.

Johnson, P. T. J., K. B. Lunde, R. W. Haight, J. Bowerman \& A. R. Blaustein. 2001. Ribeiroia ondatrae (Trematoda: Digenea) infection induces severe limb malformations in western toads (Bufo boreas). Canadian Journal of Zoology, 79: 370-379.

Johnson, P. T. J., K. B. Lunde, E. M. Thurman, E. G. Ritchie, S. N. Wray, D. R. Sutherland, J. M. Kapfer, T. J. Frest, J. Bowerman, \& A. R. Blaustein. 2002. Parasite (Ribeiroia ondatrae) infection linked to amphibian malformations in the western United States. Ecological Monographs, 72: $151-168$.

Meteyer, C. U. 2000. Field guide to malformations of frogs and toads with radiographic interpretatio$n s$. Biological Science Report USGS/BRD/BSR-2000-0005.

Meteyer, C. U., I. K. Loeffler, J. F. Fallon, K. A. Converse, E. Green, J. C. Helgen, S. Kersten, R. Levey, L. Eaton-Poole \& J. G. Burkhart. 2000. Hind Limb Malformations in Free-Living Northern Leopard Frogs (Rana pipiens) from Maine, Minnesota, and Vermont Suggest Multiple Etiologies. Teratology, 62: 151-171.

Piha, H. H., M. Pekkonen \& J. Merilä. 2006. Morphological Abnormalities in Amphibians in Agricultural Habitats: A Case Study of the Common Frog Rana temporaria. Copeia, 4: 810-817.

Rajakaruna, R. S., P. M. J. R. Piyatissa, U. A. Jayawardena, A. N. Navaratne \& P. H. Amerasinghe. 2008. Trematode infection induced malformations in the common hourglass treefrogs. Journal of Zoology, 275: 89-95.

Reyna-Bustos, O. F., I. T. Ahumada-Carrillo \& O. Vázquez-Huízar. 2007. Anfibios y reptiles del bosque La Primavera: guía ilustrada. Universidad de Guadalajara y Gobierno del Estado de Jalisco. Guadalajara, Jalisco, México.

Robles-Mendoza, C., C. García-Basilio, S. Cram-Heydrich, M. Hernández-Quiroz \& C. VanegasPérez. 2009. Organophosphorus pesticides effect on early stages of the axolotl Ambystoma mexicanum (Amphibia: Caudata). Chemosphere, 74: 703-710.

SEMARNAT. 2010. Norma Oficial Mexicana NOM-059-ECOL-2001. Protección ambiental. Especies nativas de México de flora y fauna silvestres. Categorías de riesgo y especificaciones para su 
inclusión, exclusión o cambio. Lista de especies en riesgo. Diario Oficial de la Federación, 30 de Diciembre de 2010.

Thomson Reuters. 2010. ISI Web of Science. http://apps.isiknowledge.com.wdg.biblio.udg.mx. (Consultado el 4 de Octubre 2010).

Vázquez-Díaz, J. \& G. E. Quintero-Díaz. 2005. Anfibios y reptiles de Aguascalientes. CONABIO/ CIEMA, A.C. México.

Williams, R. N., D. H. Bos, D. Gopurenko \& J. A. DeWoody. 2008. Amphibian malformations and inbreeding. Biology letters, 4: 549-552.

\section{José Luis Barragán-Ramírez \& José Luis NavarRete-Heredia}

Centro de Estudios en Zoología,

Universidad de Guadalajara,

Apdo. Postal 134, Zapopan, Jalisco, México.

<barragan5478@yahoo.com.mx> 